Giodana Borges de Holanda

\title{
DO SUBLIME TECNOLÓGICO ÀS CARTOGRAFIAS DOS FLUXOS
}

Tese apresentada ao Programa de Pósgraduação em Design da PUC-Rio como requisito parcial para obtenção do título de Doutor em Design

Orientadora: Rejane Spitz 
Giodana Borges de Holanda

\title{
DO SUBLIME TECNOLÓGICO ÀS CARTOGRAFIAS DOS FLUXOS
}

Tese apresentada como requisito parcial para obtenção do grau de Doutor pelo Programa de Pós-Graduação em Design do Departamento de Artes \& Design do Centro de Teologia e Ciências Humanas. Aprovada pela Comissão Examinadora abaixo assinada.

\author{
Profa. Rejane Spitz \\ Orientadora \\ PUC-Rio \\ Profa. Rita Maria de Souza Couto \\ PUC-Rio \\ Profa. Tamara Tânia Cohen Egler \\ UFRJ \\ Profa. Lúcia Isaltina Clemente Leão \\ PUC-SP \\ Profa. Ana Maria Nicolaci da Costa \\ PUC-Rio \\ Prof. Paulo Fernando Carneiro de Andrade \\ Coordenador Setorial do Centro de Teologia \\ e Ciências Humanas - PUC-Rio
}


Todos os direitos reservados. É proibida a reprodução total ou parcial do trabalho sem autorização da universidade, da autora, ou da orientadora.

\section{Giodana Borges de Holanda}

Artista plástica. Graduada em Arquitetura e Urbanismo pela Universidade Federal de Pernambuco em 1975. Mestre em Design pela PUC-Rio em 2002. Estudou Artes na década de 1980 em cursos livres e ateliês no CCE da PUC-Rio, na Escola de Artes Visuais do Parque Lage, e na Universidade da Califórnia em Berkeley. Trabalhando na área gráfica (desenho, gravuras, fotografia e design gráfico) na década de 1980 e em arte eletrônica a partir do final dos anos 1990, participou de diversas exposições individuais e coletivas. É professora na Escola de Artes Visuais do Parque Lage, Rio de Janeiro desde 1985, onde atua no Laboratório de Arte e Tecnologia

Ficha Catalográfica

Holanda, Giodana Borges de

Do sublime tecnológico às cartografias de fluxos / Giodana Borges de Holanda; orientadora: Rejane Spitz. - 2008.

155 f. : il. (col.) ; $30 \mathrm{~cm}$

Tese (Doutorado em Artes e Design)-Pontifícia Universidade Católica do Rio de Janeiro, Rio de Janeiro, 2008.

Inclui bibliografia

1. Artes - Teses. 2. Arte colaborativa. 3. Espaços de fluxos. 4. Conectividade. 5. Mobilidade. 6. Vida cotidiana. 7. Sublime tecnológico. 8. Estética dos fluxos. 9. Cartografias dos fluxos. I. Spitz, Rejane. II. Pontifícia Universidade Católica do Rio de Janeiro. Departamento de Artes e Design. III. Título. 
Para Geovan, amor da minha vida, com quem compartilho o cotidiano.

Para Adriano, meu filho querido.

Para minha querida mãe Luzia. 


\section{Agradecimentos}

À minha orientadora Professora Rejane Spitz, pelo incentivo constante e dedicação ao longo desses quatro anos de convívio, pela parceria para a realização deste trabalho, pela amizade e carinho.

À CAPES e à PUC-Rio, pelos auxílios concedidos, sem os quais este trabalho não poderia ter sido realizado.

Aos professores do Departamento de Artes e Design, pelos bons cursos e contribuições, especialmente à Professora Rita Couto pelo bom convívio e ensinamentos.

À Professora Ana Maria Nicolaci-da-Costa do Departamento de Psicologia da PUC-Rio, pelos cursos e valorosas contribuições.

Aos professores que participaram da Comissão examinadora.

Aos meus colegas da PUC-Rio, especialmente aos do LAE/NAE-Laboratório de Arte Eletrônica da PUC-Rio, colegas de orientação, pelas trocas, por me manter informada sobre outras áreas de trabalho.

Ao Geovan, meu marido, por ter sempre me incentivado a cursar o doutorado, e especialmente, pelo carinho e paciência em todas as horas.

Ao Adriano, meu filho, pela paciência nos momentos finais.

Aos meus pais, pelo carinho e educação que me proporcionaram.

Aos amigos e amigas, especialmente, Bia Amaral e Tina Velho, pelo carinho e amizade, pelo incentivo, e pela paciência por minha ausência nos projetos do NAT_EAV-Núcleo de Arte e Tecnologia da Escola de Artes Visuais, onde trabalhamos.

À amiga Maria Tornaghi, pelo carinho e pelas colaborações.

Aos funcionários do Departamento de Artes e Design, pela eficiência em seus trabalhos. 


\section{Resumo}

Holanda, Giodana; Spitz, Rejane. Do sublime tecnológico às cartografias dos fluxos. Rio de Janeiro, 2008. 155p. Tese de Doutorado - Departamento de Artes e Design, Pontifícia Universidade Católica do Rio de Janeiro.

Um número crescente de artistas e designers produzem na atualidade trabalhos artísticos interativos, munidos de dispositivos móveis de comunicação sem fio, como telefones celulares, PDAs, laptops, em conexões a redes computacionais e serviços de localização. Esses trabalhos mapeiam novas formas de fluxo que, de forma invisível, se acumulam em camadas espaciais do nosso viver tecnológico, revelando uma nova configuração urbana - a cidade conectada - um novo conceito de cidade que associa as práticas do cotidiano a processos colaborativos criados pelas redes de comunicação e sistemas de localização. Apresentamos alguns desses trabalhos evidenciando duas abordagens artísticas. Uma, mostrada pelo viés do sublime tecnológico, dando ênfase à presença da tecnologia em nossa vida como um fenômeno da ordem do sublime, de grandeza e poder incomensuráveis que transcendem a capacidade de apreensão humana, mas que pela própria tecnologia é desvelado. Outra, pela ótica de uma nova relação entre arte e vida cotidiana, expressa em formas de um nomadismo urbano conectado que denominamos cartografias dos fluxos, que constituem mapeamentos de percursos e derivas pela cidade, em conexão a redes e sistemas de localização. As duas abordagens se entrelaçam em uma questão central, que é o caráter colaborativo da criação artística, configurando, em ambos os casos, uma estética do fluxo. Dessas ações coletivas, que envolvem artistas e participantes, resulta uma cartografia do hibridismo espacial dos fluxos em redes e territórios em que vivemos - subjetiva e emocional, pessoal e social: uma cartografia da vida cotidiana em fluxo, uma cartografia sublime. 


\section{Palavras-chave}

Arte colaborativa, espaços de fluxos, conectividade, mobilidade, vida cotidiana, sublime tecnológico, estética dos fluxos, cartografias dos fluxos. 


\section{Abstract}

Holanda, Giodana; Spitz, Rejane.(Advisor) From the Technological Sublime to the Flow Cartographies. Rio de Janeiro, 2008. 155p. Ph.D. Thesis - Departamento de Artes e Design, Pontifícia Universidade Católica do Rio de Janeiro.

Recently, a growing number of artists and designers are producing interactive artworks equipped with mobile and wireless communications devices, including mobile phones, PDAs and laptops, connected to computer networks and location-based systems. These artworks are mapping new forms of flow that accumulate invisibly in space in layers of our technological living, and are bringing to light a new urban configuration - the wired city. This new concept of city relates everyday actions to collaborative processes created by networked mobile communications and locative media. We present some of these works from two aesthetic standpoints, the first of which is technological sublime. This emphasizes the presence of technology in our lives as a phenomenon on the order of the sublime, of immeasurable magnitude and power, that transcends the realm of human apprehension and is unveiled only by the technology itself. The second reflects a new relation between art and everyday life expressed in the form of a wired urban nomadism. We call it flow cartography - the mapping of pathways and drifts through the city connected with networks and location systems. The two approaches intertwine in a key question: Is the collaborative nature of the artistic creation resulting, in both cases, in an aesthetic of flow? These collective actions involving artists and participants result in a cartography of the hybrid space of flows in the - subjective and emotional, personal and social - networks and territories we live in: a cartography of the everyday flow of life, a sublime cartography. 
Keywords

Collaborative art, space of flows, connectivity, mobility, everyday life, technological sublime, aesthetics of flows, flow cartographies.

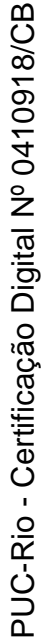




\section{Sumário}

$\begin{array}{ll}\text { 1. Introdução } & 14\end{array}$

2. Metodologia 22

3. Cotidiano em fluxo 28

3.1. Fluxos 28

3.1.1. Fixos e fluxos $\quad 28$

3.1.2. Fluidez pós-moderna 30

3.1.3. Nômades em redes 33

3.2. Espaços 36

3.2.1. Espaço de fluxos 36

3.2.2. Do lugar ao ciberespaço 38

3.2.3. Do ciberespaço ao espaço híbrido 40

3.2.4. Cidades fluidas 42

3.3. Cotidiano 46

3.3.1. Cotidiano e práticas cotidianas 46

3.3.2. Cotidiano em fluxo 48

3.3.3. Poéticas de um novo cotidiano 49

4. Sublime tecnológico 50

4.1. Sublime dos fluxos 50

4.1.1. Estética das comunicações $\quad 50$

4.1.2. Origens do Sublime 53

4.1.3. Sublime tecnológico $\quad 54$

4.1.4. O sublime dos fluxos 56

4.1.4.1. Sky Ear 58 
4.1.4.3. Dialtones - A Telesymphony 68

4.1.4.4. Electrical Walks 72

4.1.5. Sublime que conecta pessoas 75

5. Cartografias dos fluxos 77

5.1. Mapeamentos e visualizações 77

5.1.1. Cartografias híbridas $\quad 77$

5.1.2. Mapas do território 78

5.1.3. Mapas e imagens do mundo 81

5.1.4. Mapear fluxos 83

5.1.5. Mapear fluxos nas redes $\quad 84$

5.1.6. Mapeamentos das interações $\quad 89$

5.1.7. Mapear fluxos no cotidiano 93

5.1.8. Design dos processos e fluxos 97

5.1.8.1. Mobile Landscape - Graz in Real Time 100

5.1.8.2. Real Time Rome 103

5.2. Cartografias dos fluxos 106

5.2.1. Arte da mobilidade: estética do fluxo 106

5.2.2. Percursos e mapeamentos 107

5.2.3. Práticas cotidianas na cibercidade 110

5.2.4. As Cartografias dos fluxos 111

5.2.4.1. GPS Drawings $\quad 112$

5.2.4.2. Amsterdam Real Time - Diary in traces $\quad 115$

5.2.4.3. Choreography of Everyday Movement 118

5.2.4.4. The Walking Project 121

5.2.4.5. Urban Tapestries 125

5.2.4.6. Bio Mapping 131

5.2.5. Cartografias pessoais e colaborativas 136

6. Considerações finais 138

7. Referências bibliográficas 148 


\section{Lista de figuras}

Figura 1 - GPS Drawings (Jeremy Wood \& Hugh Pryor) 15

Figura 2 - Visualização da rede NSFNET (D.Cox e R.Patterson) 18

Figura 3 - Sky Ear (Usman Haque) 59

Figura 4 - Sky Ear (Usman Haque) 60

Figura 5 - Sky Ear (Usman Haque) 62

Figura 6 - Sky Ear (Usman Haque) 62

Figura 7 - Amodal Suspension (Rafael Lozano-Hemmer) 63

Figura 8 - Amodal Suspension (Rafael Lozano-Hemmer) 64

Figura 9 - Amodal Suspension (Rafael Lozano-Hemmer) 65

Figura 10 - Amodal Suspension (Rafael Lozano-Hemmer) 65

Figura 11 - Amodal Suspension (Rafael Lozano-Hemmer) 66

Figura 12 - Dialtones - a telesymphony (Golan Levin et al.) 69

Figura 13 - Dialtones - a telesymphony (Golan Levin et al.) 69

Figura 14 - Dialtones - a telesymphony (Golan Levin et al.) 70

Figura 15 - Electrical Walks (Christina Kubisch) 72

Figura 16 - Electrical Walks (Christina Kubisch) 73

Figura 17 - Electrical Walks (Christina Kubisch) 74

Figura 18 - Visualização da Internet (CAIDA) 86

Figura 19 - Visualização da Internet (Philippe Bourcier) 87

Figura 20 - Visualização da rede NSFNET (D.Cox e R.Patterson) 88

Figura 21 - Mapa da Blogosfera (Matthew Hurst) 89

Figura 22 - Mapa do Flickr (Bia Amaral) 91

Figura 23 - Mapa do Flickr com geoetiquetas 91

Figura 24 - Visualização de redes no Flickr (Gustavo G.) 92

Figura 25 - Visualização de redes no Flickr (Gustavo G.) 92

Figura 26 - Visualização de redes no Flickr (Fabien Girardin) 93

Figura 27 - Mapa situacionista $\quad 95$

Figura 28 - Mapa situacionista 96

Figura 29 - Graz in Real Time (SENSEable City Lab e Carlo Ratti) 101

Figura 30 - Graz in Real Time (SENSEable City Lab e Carlo Ratti) 102 
Figura 31 - Real-TimeRome (SENSEable City Lab e Carlo Ratti) 103

Figura 32 - Real-TimeRome (SENSEable City Lab e Carlo Ratti) 104

Figura 33 - Real-TimeRome (SENSEable City Lab e Carlo Ratti) 105

Figura 34 - GPS Drawings (Jeremy Wood \& Hugh Pryor) 113

Figura 35 - GPS Drawings (Jeremy Wood \& Hugh Pryor) 114

Figura 36 - GPS Drawings (Jeremy Wood \& Hugh Pryor) 115

Figura 37 - Amsterdam Realtime (Esther Polak e Waag Society) 116

Figura 38 - Amsterdam Realtime (Esther Polak e Waag Society) 116

Figura 39 - Amsterdam Realtime (Esther Polak e Waag Society) 117

Figura 40 - The Choreography of Everyday Movement (Teri Rueb) 119

Figura 41 - The Choreography of Everyday Movement (Teri Rueb) 119

Figura 42 - The Walking Project (Block, Ramsden e Walk \& Squawk) 121

Figura 43 - The Walking Project (Block, Ramsden e Walk \& Squawk) 122

Figura 44 - The Walking Project (Block, Ramsden e Walk \& Squawk) 124

Figura 45 - Urban Tapestries (Giles Lane, Alice Angus e Proboscis) 125

Figura 46 - Urban Tapestries (Giles Lane, Alice Angus e Proboscis) 126

Figura 47 - Urban Tapestries (Giles Lane, Alice Angus e Proboscis) 127

Figura 48 - Urban Tapestries (Giles Lane, Alice Angus e Proboscis) 127

Figura 49 - Urban Tapestries (Giles Lane, Alice Angus e Proboscis) 129

Figura 50 - Urban Tapestries (Giles Lane, Alice Angus e Proboscis) 130

Figura 51 - Urban Tapestries (Giles Lane, Alice Angus e Proboscis) 130

Figura 52 - Bio Mapping (Christian Nold) 131

Figura 53 - Bio Mapping (Christian Nold) 132

Figura 54 - Bio Mapping (Christian Nold) 132

Figura 55 - Bio Mapping (Christian Nold) 133

Figura 56 - Bio Mapping (Christian Nold) 134

Figura 57 - Bio Mapping (Christian Nold) 134

\section{Gráficos}

